\title{
Modified Robust Technique for Intra and Inter Constraint based on Video Enhancement using Colour Modeling of Countable Frames
}

\author{
Shaik. Abdul Masood ${ }^{1}$ and Shaik Mahaboob Basha ${ }^{2}$ \\ ${ }^{1}$ Post Graduate Student, \\ Department of Electronics and Communication Engineering, \\ Priyadarshini College of Engineering and Technology, Andhra Pradesh, India \\ ${ }^{2}$ Associate Professor and Head of the Department, \\ Department of Electronics and Communication Engineering, \\ Priyadarshini College of Engineering and Technology, Andhra Pradesh, India \\ 12abdulmasood448@gmail.com, ${ }^{2}$ mohisin7@yahoo.co.in
}

\begin{abstract}
New trends are more essential in the area of video enhancement for various emerging needs. Hence, we proposed a novel technique which efficiently suited for various types videos. This paper discussed about the video enhancement aiming to achieve high intra frame and inter frame qualities in an entire video. We proposed and evaluated two parameters i.e., Intra frame quality (among the frames) of the entire video and inter frame quality (in between the frames) of the entire video. Such as multiple regions of interest that can be adaptively and simultaneously enhanced.

Two steps were incorporated in to the proposed work for object quality retrieval. These are (i) ACB step improve intra frame analysis features for region-of-interest a global tone curve is created by fusing from other region and (ii) ECB step improve inter frame quality implement by histogram equalization method. We observed better improvement in PSNR values and considerable reduction in MSE values over the existing techniques. Thus, the modified technique improves the video enhancement with respect to the countable number of frames and valid better results at various levels of frames. We also evaluated the execution time in the process of video enhancement which is the ultimate parameter to discuss the power consumption. The observations of the proposed technique surpass the existing methods in the area of video enhancement in frame by frame procedure. The results of the proposed technique outperform present techniques and werre simulated using MATLAB.
\end{abstract}

Key words: Intra frame, inter frame, video enhancement, peak signal to noise ratio and mean square error, execution time

\section{Introduction}

Video enhancement plays on various types such as entrainment, healthcare, communication and surveillance. Such as maintain the quality of video and images from various parameters like video surveillance cameras, medical imaging, and also different type of frames are in poor quality. The quality of video takes different number of frames in to applied enhancement and region of interest and also applied for calculating the Peak Signal to Noise Ratio (PSNR). Thus a good enhancement video can be delivered. 


\subsection{Multiple Region of Interest (ROIs) with Single Frame Maintain Quality Enhancement}

A region of interest is a portion of an image that you want to filter or perform some other operation on. You define an ROI by creating a binary mask, which is a binary image that is the same size as the image you want to process with pixels that define the ROI set to 1 and all other pixels set to 0 . The intra frame quality of a image based on some predefined global metrics Such PSNR and Mean Square Error (MSE).

\section{Literature Survey on Existing Systems}

The existing enhancement algorithms only focus on improving the intra frame qualities within a single frame or an image. They are not suitable for enhancing videos since the inter frame quality consistencies among frames are not considered. Some state-of-the-art algorithms can be extended for enhancing inter frame qualities under some specific applications. The intra-frame quality of a picture based on some pre-defined global metrics. Since these methods do not consider region differences within an image, they cannot guarantee all the important regions inside the image will be enhanced properly.

Several contrast enhancement techniques have been introduced to improve the contrast of an image. These techniques can be broadly categorized into two groups: direct methods and indirect methods. Direct methods define a contrast measure and try to improve it. Indirect methods, on the other hand, improve the contrast through exploiting the under-utilized regions of the dynamic range without defining a specific contrast term. Since these methods do not consider region differences within an image, they cannot guarantee all the important regions inside the image will be enhanced properly.

\subsection{Among the Fames of Inter Frame Quality Enhancement}

By using the start of the art algorithm only focus on improving the single frame quality or on image. Improve the brightness and colour modeling of some specific regions.

Other approaches, since color modeling is more subjective than brightness and contrast, it is difficult to come up with a quantitative formula to define what a visually appealing color modeling is. Therefore, existing approaches on example-based image enhancement often requires a user to select an example image. The image processing techniques being developed that improve the brightness and contrast of the captured images Exposure, however, is not the only affects the perceptual quality of a mobile cam video.

\subsubsection{Colour Modeling Transfer to Image:}

Our goal is to manipulate RGB images, which are often of unknown phosphor chromaticity; we first show a reasonable method of converting RGB signals to Ruderman et al.'s perception-based colour space $1 \alpha \beta$. Because $1 \alpha \beta$ is a transform of LMS cone space, we first convert the image to LMS space in two steps. The first is a conversion from RGB to $\mathrm{XYZ}$ tri-stimulus values. This conversion depends on the phosphors of the monitor that the image was originally intended to be displayed on. Because that information is rarely available, we use a device-independent conversion that maps white in the chromaticity diagram (CIE xy) to white in RGB space. We can imagine many methods for applying the colours of one image to another. Our goal is to do so with a simple algorithm, and our core strategy is to choose a suitable colour space and then to apply simple operations there. When a typical three channel image is represented in any of the most well-known colour spaces, there will be correlations between the different channels' values. 
For example, in RGB space, most pixels will have large values for the red and green channel if the blue channel is large. This implies that if we want to change the appearance of a pixel's colour in a coherent way, we must modify all colour channels in tandem. This complicates any colour modification process. What we want is an orthogonal colour space without correlations between the axes.

\subsubsection{Contrast Enhancement:}

The aforementioned contrast enhancement techniques perform well on some images but they can create problems when a sequence of images is enhanced, or when the histogram has spikes, or when a natural looking enhanced image is strictly required. In addition, computational complexity and controllability become an important issue when the goal is to design a contrast enhancement algorithm for consumer products. In summary, our goal in this paper is to obtain a visually pleasing enhancement method that has low-computational complexity and can be easily implemented on FPGAs or ASICs and works well with both video and still images.

\section{Proposed System and Architecture}

A new intra-and-inter-constraint-based $(\mathrm{A}+\mathrm{ECB})$ algorithm is proposed. The proposed algorithm analyzes features from different ROIs and creates a "global" tone mapping curve for the entire frame such that different regions inside a frame can be suitably enhanced at the same time. Furthermore, new inter frame constraints are introduced in the proposed algorithm to further improve the inter frame qualities among frames. We will discuss the idea of our algorithm based on the histogram equalization modification based (HEM) method, we will use boldface to represent vectors and use lightface to represent scalars and functions.

Frame may contain multiple ROIs; it is desirable for the enhancement algorithm to achieve high intra frame quality of the entire picture where multiple ROIs can be adaptively and simultaneously enhanced.

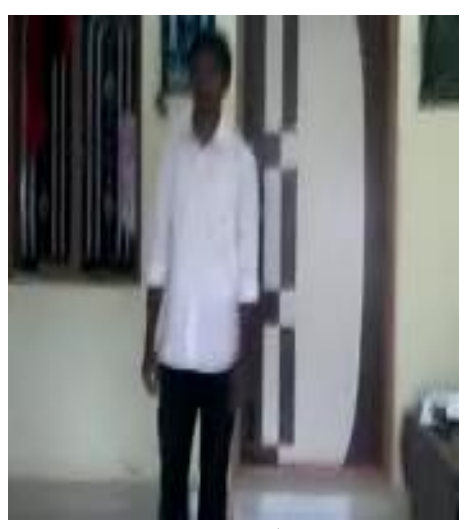

Frame1

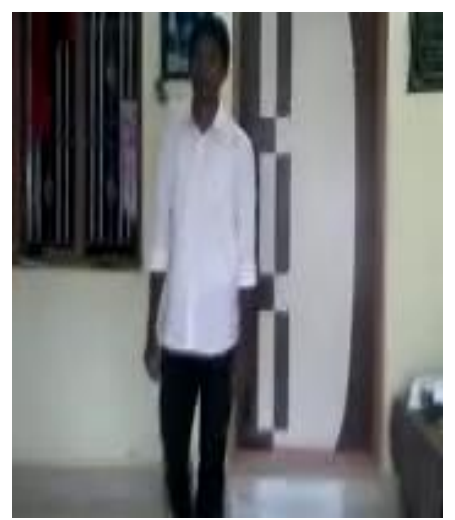

Frame10 


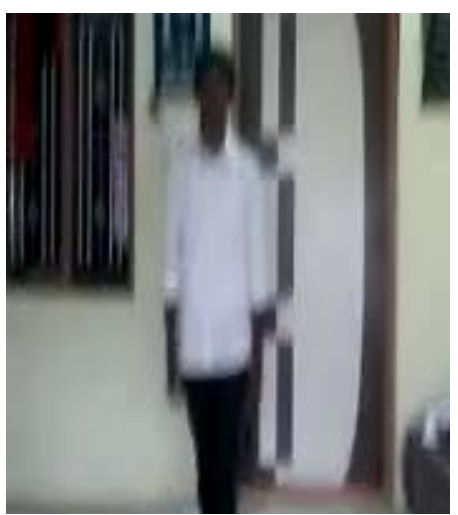

Frame11

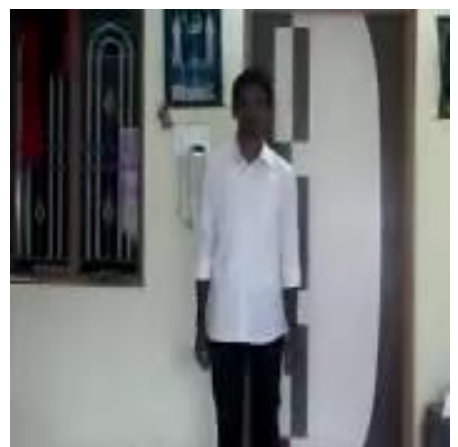

Frame 21

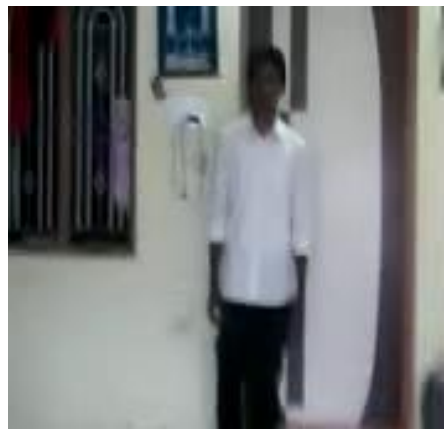

Frame31

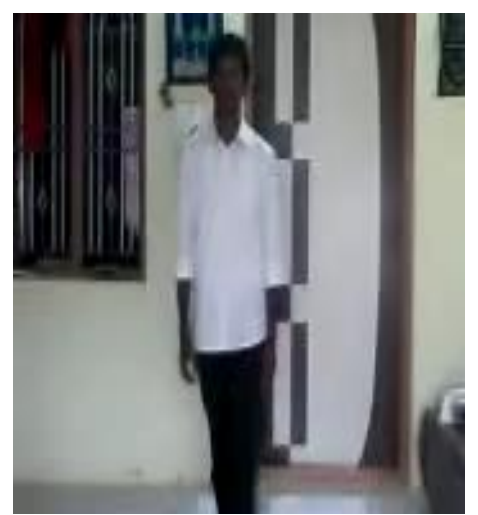

Frame20

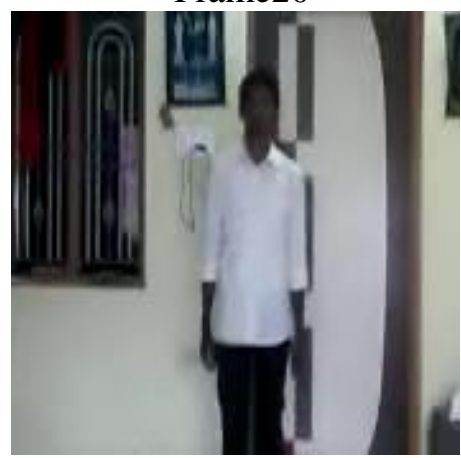

Frame30

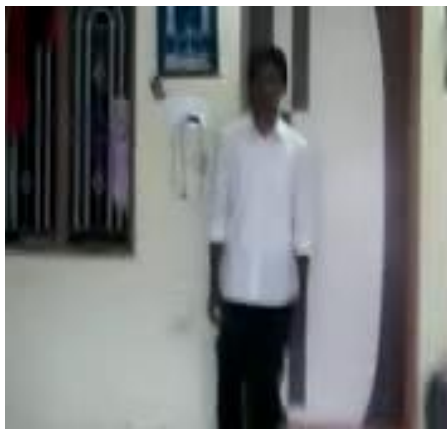

Frame40

Figure 1. Original Video Stream of Frames

We observed better improvement in PSNR values and considerable reduction in MSE values over the existing techniques. Thus, the modified technique improves the video enhancement with respect to the countable number of frames and valid better results at various levels of frames.

\section{Block Digram of the Proposed Technique}

We proposed a novel technique to improve the inter frame quality and intra frame quality on the basis of $(\mathrm{A}+\mathrm{ECB})$ enhancement algorithm. We also evaluated the execution time in the process of video enhancement which is the ultimate parameter to discuss the power consumption.

The video stream of our A+ECB algorithm can be described as In Figure a, an input frame is first enhanced by the proposed ACB step for improving the intra frame quality. Then, the 
resulting frame will be further enhanced by the proposed ECB step for handling the inter frame constraints.

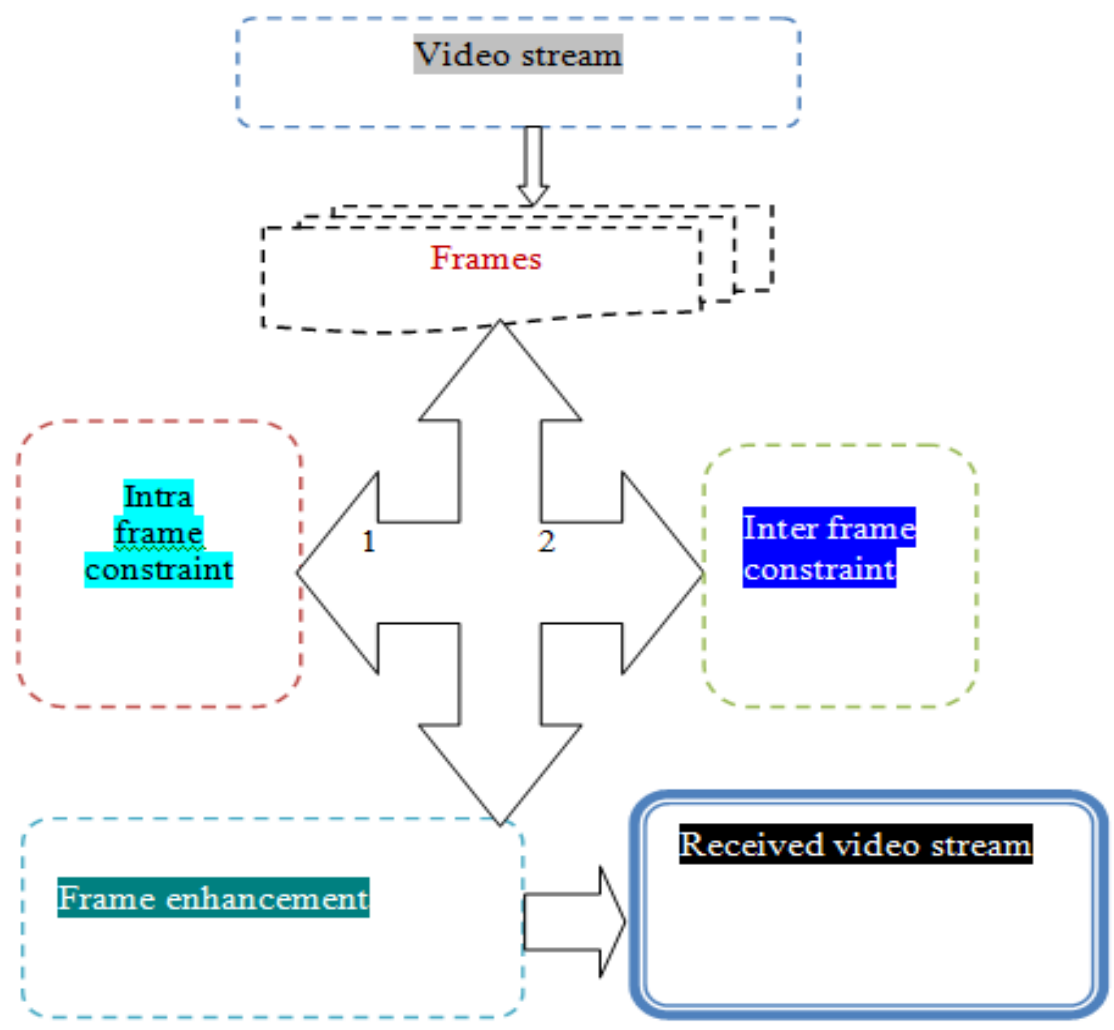

Figure 2. Block Diagram of the Proposed Technique

The simulation results followed in the next section. The figures were verified at various frames.

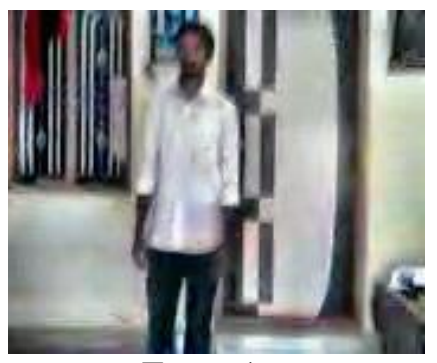

Frame1

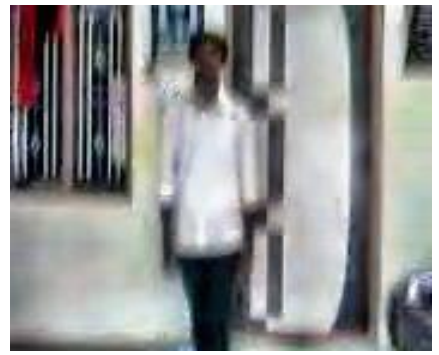

Frame11

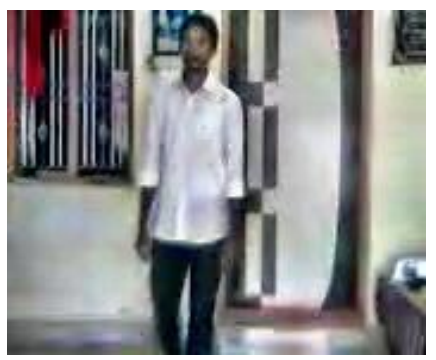

Frame10

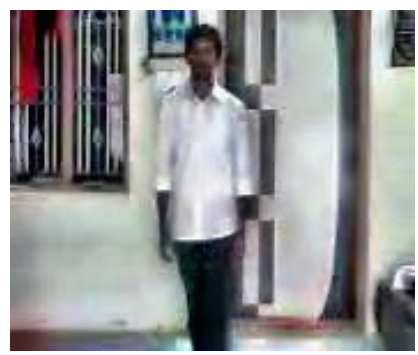

Frame20 


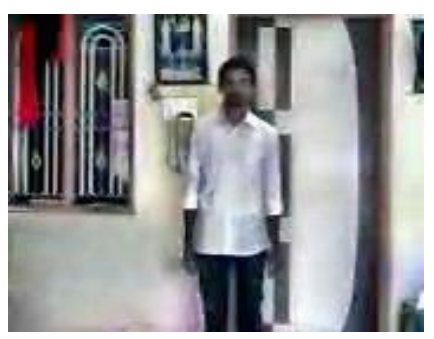

Frame21

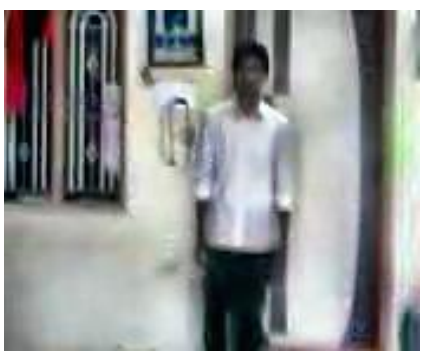

Frame31

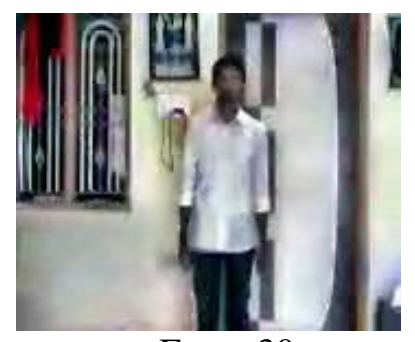

Frame30

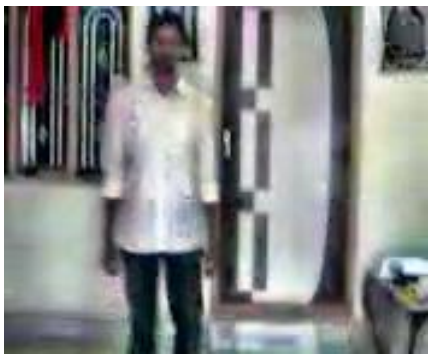

Frame40

Figure 3. Enhanced Color Modeling Frames

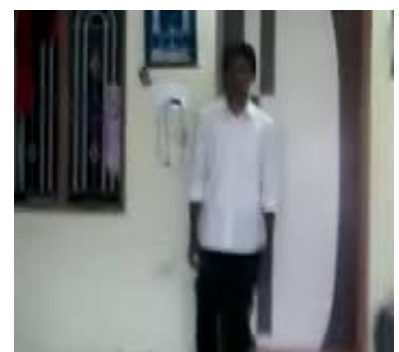

(a)

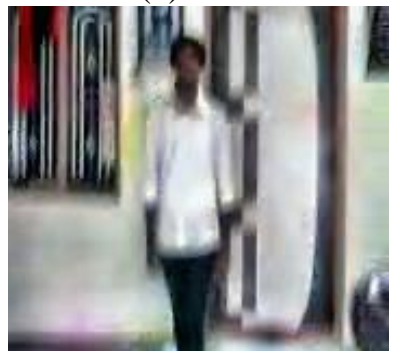

(c)

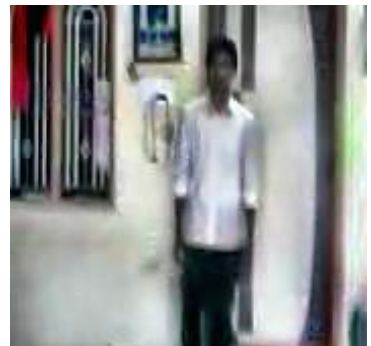

(b)

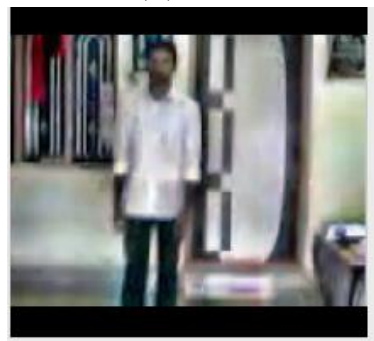

(d)

Figure 4. (a) Original Video (b) Enhanced Image by Histogram Equalization (c) Enhanced Image Focusing on the Screen Region by Gaussian Mixture Model (d) Received Video

The video stream takes diferent frames. At first enhanced by the proposed ACB step for improving the intra frame quality constraint. Then, the resulting frame will be further enhanced by the proposed ECB step for handling the inter frame constraint. We considered a poor video divided in to the number of frames. Then each frame will be enhanced. For the quality purpose some regions are appearing not good. For example face regions are maintains the bad quality. 


\subsection{Peak Signal to Noise Ratio (PSNR)\& Mean Square Error (MSE)}

It is an expression for the ratio between the maximum possible value (power) of a signal and the power of distorting noise that affects the quality of its representation. Different image enhancement algorithms were compared systematically to identify whether a particular algorithm produces better results and PSNR stands between 40-80 dB.

The mathematical representation of the PSNR is as follows:

$$
P S N R=20 \log _{10}\left(\frac{M A X_{f}}{\sqrt{M S E}}\right)
$$

where the MSE (Mean Squared Error) is:

$$
M S E=\frac{1}{m n} \sum_{0}^{m-1} \sum_{0}^{n-1}\|f(i, j)-g(i, j)\|^{2}
$$

This can also be represented in a text based format as follows.

$\operatorname{MSE}=\left(1 /\left(m^{*} \mathrm{n}\right)\right)^{*} \operatorname{sum}\left(\operatorname{sum}\left((\mathrm{f}-\mathrm{g}) .^{\wedge} 2\right)\right)$

PSNR $=20 * \log (\max (\max (\mathrm{f}))) /\left((\mathrm{MSE})^{\wedge} 0.5\right)$

' $\mathrm{f}$ ' is matrix data of our original image ' $\mathrm{g}$ ' is the matrix data of our degraded image in question ' $\mathrm{m}$ ' is the numbers of rows of pixels of the images and ' $\mathrm{i}$ ' represents the index of that row, ' $\mathrm{n}$ ' represents the number of columns of pixels of the image and ' $\mathrm{j}$ ' represents the index. $\mathrm{MAX}_{\mathrm{f}}$ is the maximum signal value that exists in our original "known to be good" image.

\section{Simulation Results}

Our system uses only 5\% CPU time for $320 \times 240$ video with frame rate of 24 frames per second on a $3.2 \mathrm{GHz}$ frequency. The Table 1 shows the comparison between the existing technique and our proposed technique.

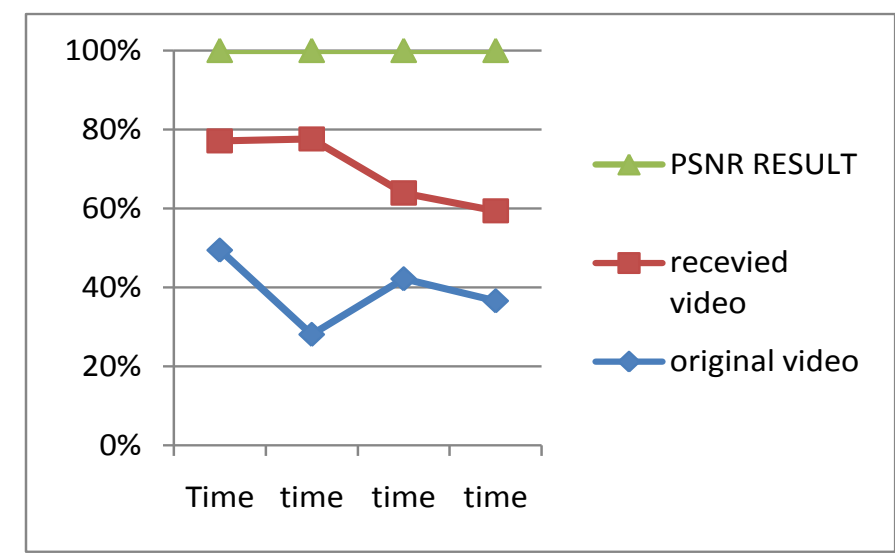

Figure 5. Performance Graph of the Proposed Work 


\section{Table 1. Comparison of PSNR Values of Existed System and the Proposed System}

\begin{tabular}{|c|c|c|}
\hline Frame No & $\begin{array}{c}\text { Existing system } \\
\text { Ref[11]'s PSNR }\end{array}$ & $\begin{array}{c}\text { Our Proposed system's } \\
\text { PSNR }\end{array}$ \\
\hline Frame 1 & $42 \mathrm{~dB}$ & $43 \mathrm{~dB}$ \\
\hline Frame 10 & $47 \mathrm{~dB}$ & $49 \mathrm{~dB}$ \\
\hline Frame 11 & $52 \mathrm{~dB}$ & $54 \mathrm{~dB}$ \\
\hline Frame 20 & $59 \mathrm{~dB}$ & $61 \mathrm{~dB}$ \\
\hline Frame 21 & $61 \mathrm{~dB}$ & $64 \mathrm{~dB}$ \\
\hline Frame 30 & $70 \mathrm{~dB}$ & $72 \mathrm{~dB}$ \\
\hline Frame 31 & $72 \mathrm{~dB}$ & $76 \mathrm{~dB}$ \\
\hline Frame 40 & $77 \mathrm{~dB}$ & $80 \mathrm{~dB}$ \\
\hline Frame 41 & $79 \mathrm{~dB}$ & $82 \mathrm{~dB}$ \\
\hline & & \\
\hline & & \\
\hline & & \\
\hline & & \\
\hline & & \\
\hline & & \\
\hline
\end{tabular}

The simulation results clearly showed the performance of the proposed new technique. The results executed on Windows 7, 2 GB RAM, 32 -bit operating system, Using MATLAB R2012a.

\section{Conclusion}

In this paper, we concluded the inter frame quality and intra frame quality of entire video stream enhanced. Different ROIs are created the entire frame such that the quality of a frame can be properly enhanced. Experimental results demonstrated the effectiveness of our algorithm. Total numbers of frames are also counted. The proposed algorithm can be validated on VLSI implementation.

\section{Acknowledgements}

I feel profoundly indebted to my guide and the Head of the Department Mr. Shaik. Mahaboob Basha for the opportunity to work with him and got benefitted from his valuable guidance and advices. It gives me immense pleasure to express my sincere thanks and gratitude to my guide Mr. Shaik. Mahaboob Basha. His inestimable help, encouragements and constructive suggestions at every stage of my research work made me to present this 
work. Further, my sincere thanks are due to the anonymous reviewers for their valuable comments to improve the final version of this paper.

\section{References}

[1] M. Sun, Z. Liu, J. Qiu, Z. Zhang and M. Sinclair, “Active lighting for video conferencing”, IEEE Trans. Circuits Syst. Video Technol., vol. 19, no. 12, (2009) December, pp. 1819-1829.

[2] T. Arici, S. Dikbas and Y. Altunbasak, "A histogram modification framework and its application for image contrast enhancement", IEEE Trans. Image Process., vol. 18, no. 9, (2009) September, pp. 1921-1935.

[3] P. Viola and M. Jones, "Robust real-time object detection", Proc. $2^{\text {nd }}$ Int. Workshop Statist. Compute. Theories Vision, (2001), pp. 137-154.

[4] Y. W. Tai, J. Jia and C. K. Tang, "Local colour transfers via probabilistic segmentation by expectationmaximization", Proc. CVPR, (2005), pp. 747-754.

[5] S. Premoze and M. Ashikhmin, "Rendering Natural Waters", Proc. Pacific Graphics, IEEE CS Press, Los Alamitos, Calif., (2000), pp. 23-30.

[6] Q. Wang and R. K. Ward, "Fast image/video contrast enhancement based on weighted thresholded histogram equalization”, IEEE Trans. Consum. Electron, vol. 53, no. 2, (2007) May, pp. 757-764.

[7] Z. Y. Chen, B. R. Abidi, D. L. Page and M. A. Abidi, "Gray-level grouping (GLG): An automatic method for optimized image contrast enhancement—Part I: The basic method", IEEE Trans. Image Process., vol. 15, no. 8, (2006) August, pp. 2290-2302.

[8] Y. W. Tai, J. Jia and C. K. Tang, "Local color transfer via probabilistic segmentation by expectationmaximization", Proc. CVPR, (2005), pp. 747-754.

[9] R. Schettini and F. Gasparini, "Contrast image correction method", J. Electron. Imaging., vol. 19, no. 2, (2010), pp. 5-16.

[10] S. Battiato and A. Bosco, "Automatic image enhancement by content dependent exposure correction," EURASIP J. Appl. Signal Process, vol. 2004, no. 12, (2004), pp. 1849-1860.

[11] A. Eden, "No-reference estimation of the coding PSNR for H.264-coded sequences", IEEE Trans. Consum. Electron, vol. 53, no. 2, (2007) May, pp. 667-674.

[12] M. A. Mofaddel and W. M. Abd-Elhafiez, "Object-based hybrid image and video coding scheme", Proceedings of International Conference Computer Engineering \& Systems, (2011), pp. 245 - 251.

\section{Authors}

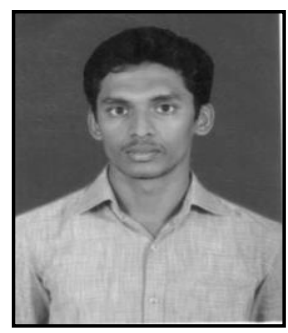

Shaik Abdul Masood, he got his Engineering graduation from J.N.T.University. Now, he is pursuing Post Graduation programM.Tech., in Priyadarshini College of Engineering, an affiliated college of the same university. He is doing his project work under the guidance of Mr. Shaik. Mahaboob Basha, Head of the Department of Electronics and Communication of Engineering.

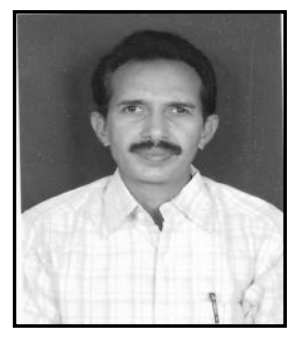

Shaik. Mahaboob Basha, he is currently working as Head of the Department of Electronics and Communication Engineering in Priyadarshini College of Engineering and Technology, Nellore, Andhra Pradesh. He is engaging with so many Engineering Professional bodies. He is a Member of IEEE, an Associate Member of The Institution of Engineers (India), and Life Member of ISOI of Indian Institute of Science, Bangalore and Member of SERSC. He is guiding several students for Under Graduate and Post Graduate programs of Engineering. He has published more than 15 papers in various National and International Journals and Conferences. 
International Journal of Signal Processing, Image Processing and Pattern Recognition Vol.7, No.4 (2014) 